Int. J. Odontostomat., 4(3):285-290, 2010.

\title{
Tratamiento Quirúrgico de Caninos Mandibulares Bilaterales Transmigrados
}

\author{
Surgical Treatment of Bilateral Mandibular Transmigrated Canine
}

\author{
Sergio Olate*; Juan Pablo Alister Herdener*; José Luis Muñante-Cárdenas ${ }^{* * *}$; \\ Leandro Pozzer $^{* * *}$ \& José Ricardo de Albergaria-Barbosa ${ }^{* * *}$
}

OLATE, S.; ALISTER, H. J. P.; MUÑANTE-CÁRDENAS, J. L.; POZZER, L. \& ALBERGARIA-BARBOSA, J. R. Tratamiento quirúrgico de caninos mandibulares bilaterales transmigrados. Int. J. Odontostomat., 4(3):285-290, 2010.

RESUMEN: La migración de caninos inferiores es un fenómeno poco común. Su incidencia es menor que incluidos maxilares y son frecuentemente encontrados en jóvenes. Solamente caninos, segundos premolares y terceros molares incluidos migran a lugares ectópicos en su desarrollo y dientes incluidos ectópicos son, en la mayoría de los casos, asintomáticos. La etiología es compleja y poco descrita en la literatura. Un canino es considerado un diente migrado cuando, horizontalmente, más de la mitad del diente atraviesa la línea mediana. Este artículo reporta un caso clínico, con tratamiento quirúrgico de exodoncia de caninos bilaterales incluidos, horizontalmente en la región de la sínfisis. Se observó que la exodoncia es la conducta operatoria más frecuente, presentando en este caso buenos resultados.

PALABRAS CLAVE: caninos transmigrados, dientes incluidos, cirugía oral.

\section{INTRODUCCIÓN}

Dientes incluidos son todos los elementos dentarios que al momento de su época normal de erupción aún permanecen inmersos en el interior de los tejidos (Gregori, 1996). Un diente no erupcionado, ocasionalmente puede migrar a un lugar distante de donde debería desarrollarse (Joshi, 2001). Cuando el diente avanza traspasando la línea mediana sin influencia de cualquier entidad patológica se denomina transmigración.

Etiología. La etiología de estas anomalías no es clara y tiene pocos relatos en la literatura internacional. Se sabe que para que ocurra la transmigración dentaria es fundamental la inclinación de la corona, sugiriendo tres hipótesis (Saad-Neto et al., 1985). La primera puede estar relacionada con el cartílago mandibular, centro de osificación mandibular, que después de inducir la formación ósea mandibular iniciada junto a la ramificación de los nervios incisivos y mentonianos se retrae formando el ligamento esfenomandibular, lo cual podría dejar un "canal residual" con menor densidad ósea o células remanecientes que favorecerían la migración dentaria.
La segunda hipótesis, aislada o asociada a la primera, seria la dirección de la osteogénesis hacia distal y mesial a partir de la ramificación de los nervios mentonianos e incisivos, y la tercera hipótesis seria la propia morfología del trabeculado óseo de la mandíbula, que podría estar asociada a las primeras, toda vez que es mantenida la integridad del capuchón pericoronario y de la superficie radicular del diente incluido. Joshi \& Shetye (1994) afirman que no es posible encontrar un factor etiológico específico o factor predisponerte para el proceso de transmigración, por lo que sugieren que una fuerza anormal de erupción puede direccionar el canino a través de la sínfisis mandibular. El mismo autor cita en el 2001 (Joshi) otros dos factores etiológicos de la migración dental. El primero de ellos seria un mal posicionamiento de la lámina dental durante la etapa de desarrollo embriológico y el segundo seria un factor genético. Camilleri \& Scerri (2003), describió caninos incluidos en la región de sínfisis asociados a lesiones quísticas, señalando también lo difícil que sería afirmar que tales lesiones fuesen causantes de la migración dentaria.

\footnotetext{
* Departamento de Odontología Integral, Facultad de Medicina, Universidad de La Frontera, Temuco, Chile.

* División de Cirugía Oral y Maxilofacial, Facultad de Odontología, Universidad Mayor, Temuco, Chile.

***ivisión de Cirugía y Traumatología Bucomaxilofacial, Facultad de Odontología de Piracicaba, Universidad Estadual de Campinas, Brasil.
} 
Incidencia. La incidencia de caninos incluidos en la mandíbula es menor que en la maxila, siendo relativamente baja. La migración dental es mas común en pacientes jóvenes, menores de 20 años. Kramer \& Williams (1970) examinaron 3745 pacientes, encontrando 48 caninos incluidos, de los cuales 3 eran mandibulares $(0,08 \%)$. Grover \& Lorton (1985) estudiaron 5000 reclutas del ejercito americano y encontraron 10979 dientes incluidos, de los cuales 153 eran caninos y solo 11 eran inferiores $(0,1 \%)$. Milano et al. (1996) reporta un estudio donde se examinaron 3000 pacientes en USA, encontrando una frecuencia de 1:20 relacionando caninos incluidos mandibulares y caninos incluidos maxilares. Saad-Neto et al. realizaron una evaluación epidemiológica en Araçatuba (SP) en 1944 pacientes, encontrando 52 caninos incluidos, de los cuales solo 3 estaban en la mandíbula, sin predilección en cuanto al sexo. Joshi presentó el año 2001 un estudio de 28 casos de transmigración mandibular de caninos, comparándolos con otros 39 casos presentados en la literatura. Observó que las mu-

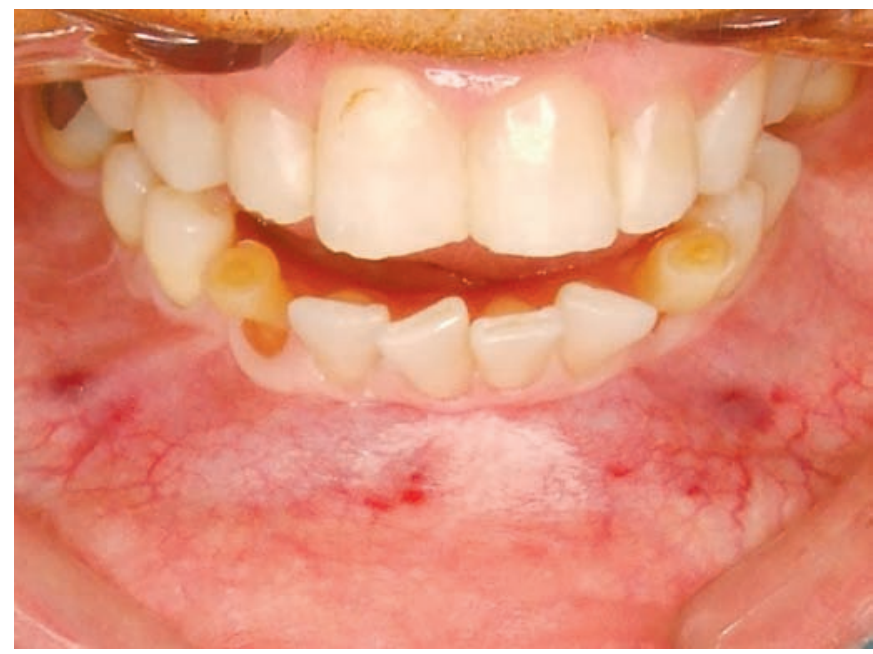

Fig. 1. Imagen clínica preoperatoria, donde es posible observar la presencia de los caninos mandibulares deciduos.

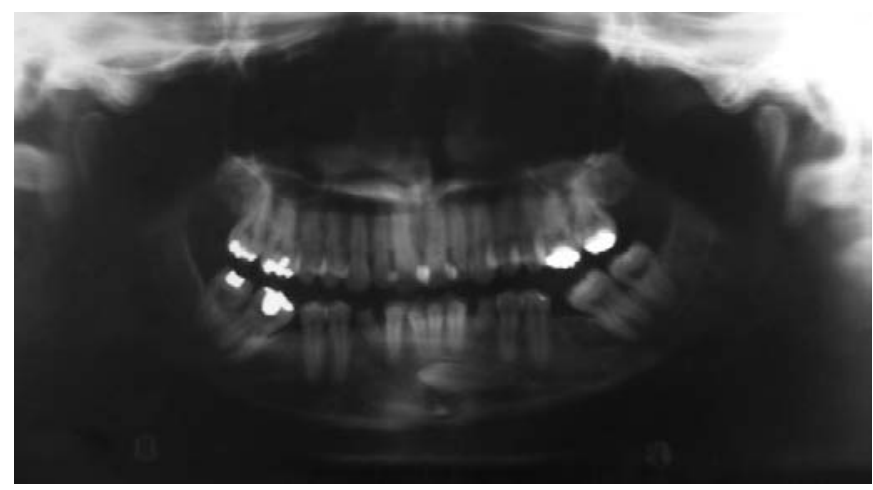

Fig. 2. Radiografia panorámica donde se observan los dientes 3.3 y 4.3 con transmigración, sobrepasando la línea media; se observa también un área radiolúcida asociada a la corona de la pieza 3.3 jeres son más afectadas; la mayoría de los casos son de migración unilateral; el canino izquierdo migra más comúnmente que el derecho; la mayoría de los casos son asintomáticos y fueron descubiertos a través de exámenes radiográficos previos a tratamientos ortodóncicos.

\section{CASO CLÍNICO}

Paciente de 36 años de edad, sexo femenino, consultó por la repentina aparición de sintomatología dolorosa e inflamatoria en relación a la zona de sínfisis y parasínfisis. Al estudio clíni$\mathrm{co}$, se evidencio una paciente clase II de Angle con ausencia de oclusión en el segmento anterior y gingivitis crónica generalizada. Se observó edentulismo parcial además de restauraciones múltiples y permanencia de los dientes 7.3 y 8.3 con evidentes signos de abrasión y atrición (Fig. 1).

Se realizaron estudios de imagenología, donde en el estudio panorámico se identificó la presencia de los dientes 3.3 y 4.3 en posición horizontal cruzando la línea mediana interincisiva junto a una reabsorción radicular de los dientes 3.2 y 3.1 ; también se observó una región radiolúcida en relación a la corona del diente 3.3 , de posición más superior que el diente 4.3 (Fig. 2). El estudio de la radiografía oclusal se identificó una posición anterior en relación al plano axial de los incisivos inferiores.

Se planificó la exodoncia de las piezas 3.3 y 4.3 con el clásico acceso intraoral para mentoplastía u obtención de injerto de mentón, con medicación preoperatorio de dexamen-tazona $4 \mathrm{mg}$ y dipirona sódica $500 \mathrm{mg}$, ambos de administración oral 1 hora antes del procedimiento quirúrgico.

Se aplicó anestesia local de lidocaina al 2\% con epinefrina de 1:100.000 para anestesia al nervio alveolar inferior bilateral y técnica infiltrativa local; se aplicó además anestesia infiltrativa de mepivecaina al $4 \%$ de forma extraoral. Se realizó una incisión mucosa y muscular en fondo de vestíbulo, con inclinación hacia el labio inferior hasta llegar a periostio. En el plano mesiodistal se descoló hasta alcanzar el nervio mentoniano y en dirección inferior hasta la base de mandíbula tanto en la región de sínfisis como en parasínfisis. Posteriormente se realizó osteotomía con fresa esférica en alta rotación identificando la región coronaria del diente 4.3 y posteriormente la región 
radicular del mismo; se realizó la odontosección y se extrajo el diente en múltiples segmentos. A continuación se realizó un procedimiento semejante sobre la región coronaria y radicular del diente 3.3 , realizando osteotomía y posterior odontosección (Fig. 3); durante la cirugía se mantuvo en posición los caninos inferiores temporales a fin de disminuir las complicaciones estéticas, considerando que en una segunda etapa se realizarían prótesis fija sobre implantes óseintegrados.
Finalmente se retiró una lesión de tejido blando en relación a la corona del diente 4.3 limitada entre la misma corona y tejido óseo circundante, clínicamente con aspecto quístico, donde al estudio histopatológico demostró ser quiste dentígero (Fig. 4). Se realizó profusa irrigación con suero fisiológico al $0,9 \%$ y limpieza con lima de hueso y cucharetas. No fue posible identificar raíces de los incisivos inferiores durante la cirugía.

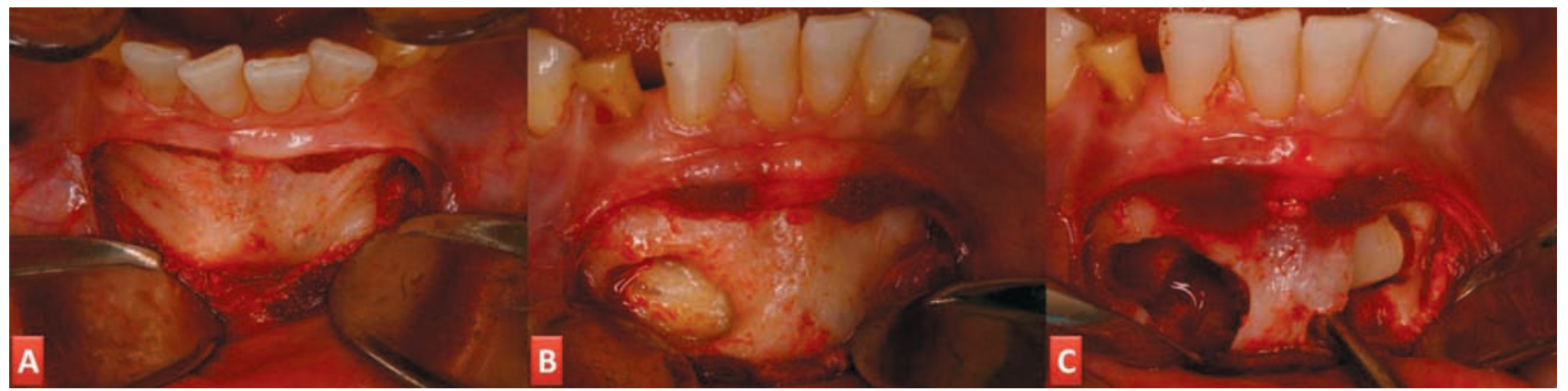

Fig. 3. Secuencia quirúrgica. A: acceso y exposición de la región de sínfisis y parasínfisis; B: osteotomía y exposición de la región coronaria del diente 4.3; C: ostectomía y exposición del diente 3.3 posterior a la extracción del diente 4.3.

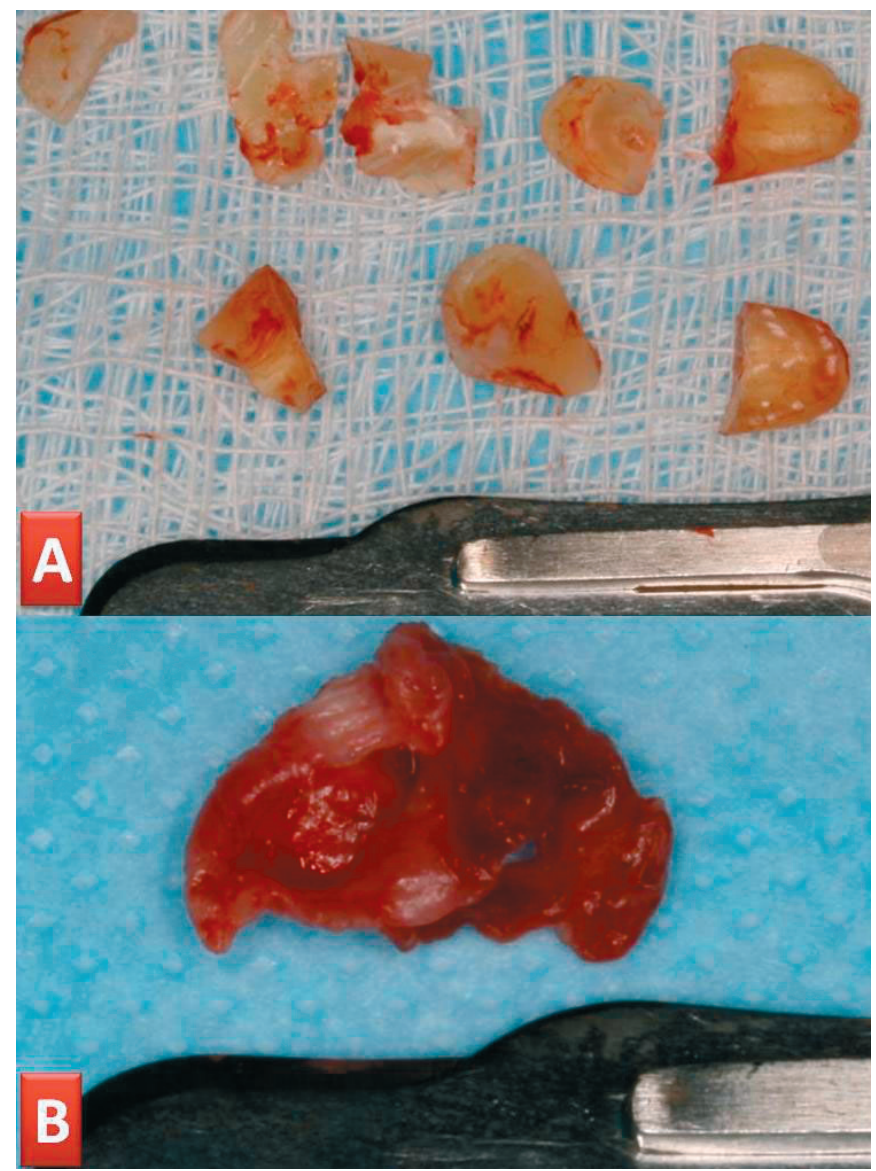

Fig. 4. Material extraído del sitio quirúrgico. A: fragmentos de los dientes 3.3 y 4.3. B: estructura quística proveniente de la región coronaria del diente 4.3 .
Se realizó la sutura del sitio quirúrgico primero con el plano muscular con sutura simple cat-gut 4-0 y finalmente de la estructura mucosa con sutura continua utilizando también cat-gut 4-0 (Fig. 5). La medicación postoperatoria fue de administración oral con dipirona sódica $500 \mathrm{mg}$ cada 4 horas por 48 horas e ibuprofeno $400 \mathrm{mg}$ cada 8 horas por 3 días. Junto a esto, la paciente realizó enjuagues con clorhexidina a partir de 24 horas posquirúrgica, mantenido durante 10 días. El primer control postoperatorio se realizó el séptimo día, evidenciando ausencia de complicaciones y buenas condiciones post-quirúrgicas y una leve parestesia regional

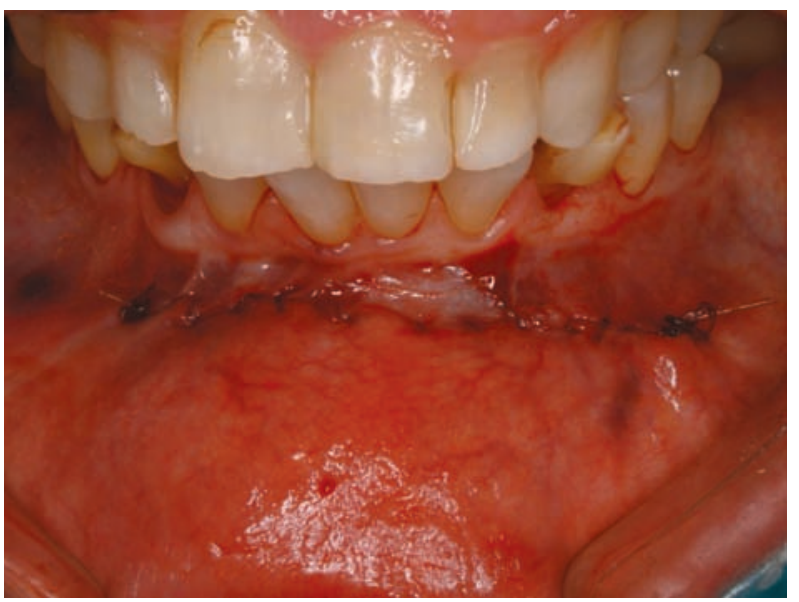

Fig. 5. Sutura por planos, comenzando por el plano muscular y finalizando por el plano mucoso con hilo absorbible cat-gut 4-0. 
en evolución favorable. Después de 2 meses de control, se observo un tejido blando completamente cicatrizado sin complicaciones que pudiesen asociarse al procedimiento quirúrgico (Fig. 6); en el estudio radiográfico también se observó un área en reparación ósea sin alteraciones en la imagen, de forma que estaba en condiciones de ser sometida a la segunda etapa quirúrgica consistente en la instalación de implantes óseintegrados (Fig. 7).

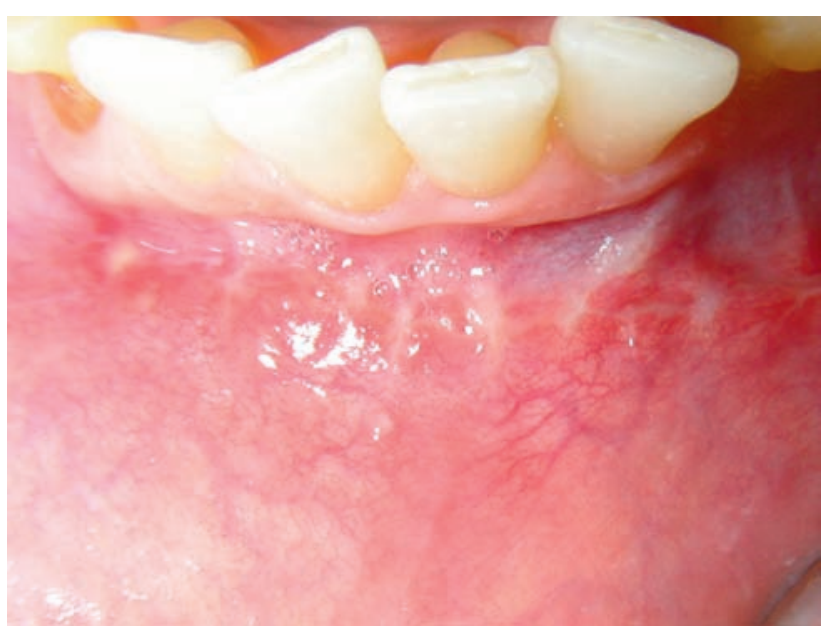

Fig. 6. Tejidos blandos en un postoperatorio de 60 días, demostrando clínicamente la cicatrización tecidual.

\section{DISCUSIÓN}

El tratamiento quirúrgico de los caninos incluidos con transmigración es citado en la literatura como el procedimiento de elección. Joshi \& Shetye afirman que la extracción se presenta como el tratamiento más favorable para caninos migrados, sin necesidad de realizar esfuerzos para traccionar el diente o devolverlo a su posición original. Wertz (1994) ya presentó el traccionamiento ortodóntico en tres casos con controversiales resultados, ya que fue necesario extraer el diente después de alcanzar un mal posicionamiento en la arcada dentaria. El autor concluye que a pesar de las grandes dificultades en el sector, es posible hacer traccionamiento en algunos casos que presenten impactación hacia vestibular.

Costello et al. (1996) afirman que se debe proceder a la exodoncia de caninos incluidos para evitar complicaciones como la reabsorción de los dientes próximos, presentando el tratamiento quirúrgico de 2 casos de caninos incluidos en mandíbula. Black \& Zallen (1973), Miranti \& Levbarg (1974), Joshi \& Shetye, Kuftinec et al. (1995) y Milano et al. relataron procedimientos quirúrgicos en pacientes de entre 14 y 23 años de edad, confirmando el grupo etario de mayor preva-

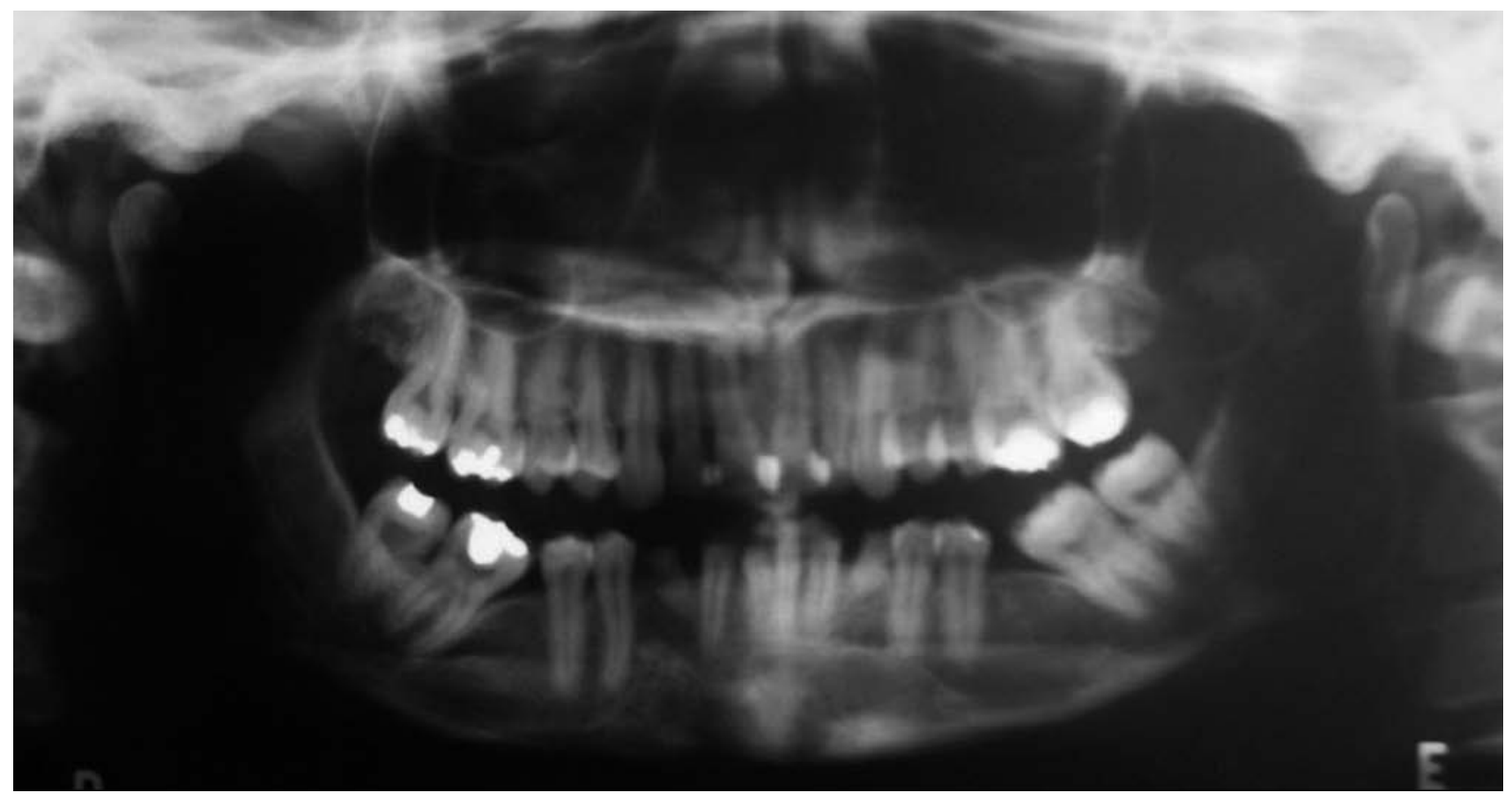

Fig. 7. Radiografía panorámica con 3 meses de postoperatorio que presenta imagen compatible con un avanzado nivel de reparación ósea, lo que permitirá iniciar la segunda etapa quirúrgica consistente en la inserción de implantes óseintegrados. 
lencia y confirmando también la exodoncia como el tratamiento de elección. Una variación a la exodoncia convencional fue relatado por Rebellato \& Schabel (2003), donde en una paciente de 12 años con exodoncia de canino inferior izquierdo incluido fue instalado en el mismo acto un implante, relatando éxito en el procedimiento. Las complicaciones postquirúrgicas se asocian con cierta frecuencia a parestesia de nervio mentoniano, pero que en la experiencia de Alaejos-Algarra et al. (1998), es solamente transitoria.
En conclusión, caninos incluidos horizontalmente en mandíbula son poco frecuentes. La etiología es parcialmente conocida y probablemente multifactorial. El diagnóstico en la mayoría de los casos es ocasional y con la ayuda de exámenes radiográficos. Es necesario hacer una planificación multidisciplinaria, donde el tratamiento quirúrgico es la opción más utilizada, presentándose como una solución rápida y eficaz tanto en el presente caso como también en los casos publicados.

OLATE, S.; ALISTER, H. J. P.; MUÑANTE-CÁRDENAS, J. L.; POZZER, L. \& ALBERGARIA-BARBOSA, J. R. Surgical treatment of bilateral mandibular transmigrated canine. Int. J. Odontostomat., 4(3):285-290, 2010.

ABSTRACT: Migration of canine mandibles is a very uncommon phenomenon. Their incidence is less frequent than maxillary canines and present frequently in young people. Only canines, second bicuspid and third molars can migrate to ectopic areas in their development and ectopic embedded teeth are, for the most part asymptomatic. The etiology is complex and with poor information. A canine is considered a migrating tooth when it penetrates horizontally the middle line of the mandible. This paper reports a case with surgical treatment of dental extraction, including horizontal bilateral canines in the symphysis region. It was observed that dental extraction is a more frequent treatment and presented with good results in this case.

KEY WORD: transmigrated canines, include teeth, oral surgery.

\section{REFERENCIAS BIBLIOGRÁFICAS}

Alaejos-Algarra, C.; Berini-Aytes, L. \& Gay-Escoda, C. Transmigration of mandibular canines: report of six cases and review of the literatura. Quintessence Int., 29(6):395-8, 1998.

Black, S. L. \& Zallen, R. D. An unusual case of tooth migration. Oral Surg. Oral Med. Oral Pathol., 36(4):607-8, 1973.

Camilleri, S. \& Scerri, E. Transmigration of mandibular canines - a review of the literature and a report of five cases. Angle Orthod., 73(6):753-62, 2003.

Costello, J. P.; Worth, J. C. \& Jones, A. G Transmigration of permanent mandibular canines. Brit. Dent. J., 181(6):212-3, 1996.

Gregori, C. Cirurgia buco-dento-alveolar. São Paulo, Ed. Sarvier, 1996.

Grover, P., S. \& Lorton, L. The Incidence of unerupted permanent teth and related clinical cases. Oral Surg. Oral Med. Oral Pathol., 59(4):420-5, 1985.

Joshi, M. R. \& Shetye, S. B. Transmigration of mandibular canines: a review of the literature and report of two cases. Quintessence Int., 25(4):2914, 1994.
Joshi, M. R. Transmigrant mandibular canines: a record of 28 cases and a retrospective review of the literature. Angle Orthod., 71(1):12-22, 2001.

Kramer, R. M. \& Williams, A. C. The incidence of impacted teeth: a survey at Harlem Hospital. Oral Surg. Oral Med. Oral Pathol., 29:237-41, 1970.

Kuftinec, M. M.; Shapira, Y. \& Nahlieli, O. A care report bilateral transmigration of impacted mandibular canines. J.A.D.A., 126:1022-3, 1995.

Milano, M.; Barrett, L. \& Marshall, E. Extraction of a horizontally impacted mandibular canine through a genioplasty approach: report of a case. J. Oral Maxillofac. Surg., 54:1240-2, 1996.

Miranti, R. \& Levbarg, M. Extraction of a horizontally transmigrated impacted mandibular canine: report of case. J.A.D.A., 88:607-10, 1974

Rebellato, J. \& Schabel, B. Treatment of patient with an impacted transmigrant mandibular canine and a palatally impacted maxillary canine. Angle Orthod., 73:328-36, 2003.

Saad-Neto, M.; de Carvalho, A.C.; Okamoto, T. \& Callestini, E. A. Impacted canines. II. Ver. Reg. 
OLATE, S.; ALISTER, H. J. P.; MUÑANTE-CÁRDENAS, J. L.; POZZER, L. \& ALBERGARIA-BARBOSA, J. R. Tratamiento quirúrgico de caninos mandibulares bilaterales transmigrados. Int. J. Odontostomat., 4(3):285-290, 2010.

Aracatuba Assoc. Paul. Cir. Dent., 6:25-31, $1985 . \quad$ Dirección para correspondencia:

Prof. Dr. Sergio Olate Morales

Wertz, R. A. Treatment of transmigrated mandibular canines. Am. J. Orthod. Dentofacial Orthop., 106(4):419-27, 1994.

Departamento de Odontología Integral

Facultad de Medicina

Universidad de La Frontera

Claro Solar 115

Temuco - CHILE

Email: solate@ufro.cl

Recibido : 13-09-2010

Aceptado: 12-11-2010 